\title{
Temporal lobe epilepsy surgery: different surgical strategies after a non-invasive diagnostic protocol
}

\author{
P P Quarato, G Di Gennaro, A Mascia, L G Grammaldo, G N Meldolesi, A Picardi, T Giampà, \\ C Falco, F Sebastiano, P Onorati, M Manfredi, G Cantore, V Esposito
}

J Neurol Neurosurg Psychiatry 2005;76:815-824. doi: 10.1136/jnnp.2004.044016

See end of article for authors' affiliations

......................

Correspondence to: Dr P P Quarato, *Epilepsy Surgery Unit, Department of Neuroscience, IRCCS "NEUROMED", 86077 Pozzilli (IS), Italy; episurg@ neuromed.it

Received 21 April 2004 Revised version received 27 August 2004

Accepted

22 September 2004

\begin{abstract}
Aim: To test a non-invasive presurgical protocol for temporal lobe epilepsy (TLE) based on "anatomoelectro-clinical correlations".

Methods: All consecutive patients with suspected TLE and seizure history $<2$ years were entered into the protocol, which included video-electroencephalographic (EEG) monitoring and magnetic resonance imaging (MRI). Three different TLE subsyndromes (mesial, lateral, mesiolateral) were identified by combined anatomical, electrical, and clinical criteria. "Tailored" surgery for each subsyndrome was offered. Patients with seizure history $<2$ years, MRI evidence of temporal mass lesion, and concordant interictal EEG and clinical data bypassed video-EEG monitoring and were directly scheduled for surgery. Results: Lesionectomy was performed without video-EEG recording in 11 patients with tumorous TLE. Of 146 patients studied with video-EEG, 133 received a TLE diagnosis. Four were excluded for neuropsychological risks, eight refused surgery, and 121 underwent surgery. Of 132 consecutive patients who underwent surgery, 101 had at least one year of follow up. They were divided into a "hippocampal sclerosis/cryptogenic" group ( $n=57$ ) and a "tumours/cortical organisation disorders" group ( $=44)$. In the first group, extensive temporal lobectomy (ETL) was performed in 40 patients, anteromesial temporal lobectomy (AMTL) in 17 patients. At follow up, 47 patients were seizure free. In the second group, lesionectomy plus ETL was performed in 23 patients, lesionectomy plus AMTL in six patients, and lesionectomy alone in 15 patients. Thirty nine patients were seizure free.

Conclusions: These findings suggest that different TLE subsyndromes can be identified accurately using non-invasive anatomo-electro-clinical data and can be treated effectively and safely with tailored surgery.
\end{abstract}

$\mathrm{D}$ rug resistant focal epilepsy is responsible for high social and economic costs in industrialised countries. ${ }^{1}$ Approximately $60 \%$ of all patients with epilepsy suffer from focal epilepsy, and in one third of these patients, most of them affected by temporal lobe epilepsy (TLE), seizures are not adequately controlled with antiepileptic drugs (AED). ${ }^{2}$ Surgery is currently accepted as an effective and safe therapeutic approach in drug resistant epilepsy, particularly in TLE, where patients become "seizure free" in 70-90\% of cases. ${ }^{3}$ However, surgery for epilepsy remains an underused, "last resort" treatment because only a small proportion of patients affected by surgically remediable epilepsies undergo this intervention. In a recent editorial, ${ }^{4}$ Engel reported fear of morbidity and confidence in new AEDs or in vagal nerve stimulation as factors that discourage patients and their physicians from surgery, which lacks sufficient data from randomised controlled trials. However, a recent randomised controlled trial of TLE surgery found it to be superior to prolonged medical treatment in terms of efficacy and safety. ${ }^{5}$ Moreover, although today there is agreement that TLE can be diagnosed in most patients without invasive tests, ${ }^{6-8}$ there is no consensus between different epilepsy surgery groups regarding the optimal use of non-invasive procedures.

The aim of an ideal non-invasive presurgical protocol in TLE surgery should be to: (1) identify the epileptogenic zone and consequently to identify potential candidates for intracranial investigations - that is, some patients affected by lateral TLE; (2) minimise the cost of human and technological resources; (3) reduce the time of diagnostic evaluation, so that surgery can be offered to more patients. In our centre, we implemented a non-invasive presurgical protocol for TLE diagnosis (TLE-Prot), based on "anatomo-electro-clinical correlations", inspired by methodological principles first proposed by Bancaud and Talairach," and later developed by their successors. ${ }^{10-12}$ This protocol allows different types of tailored resection to be offered for TLE subtypes. ${ }^{13}$

We report the results of the presurgical evaluation and the outcome of epilepsy surgery in 101 consecutive patients with TLE, diagnosed by our protocol, who have been followed for at least one year after surgery.

\section{PATIENTS AND METHODS \\ Patient population}

Between September 1999 and January 2003, 101 consecutive patients with drug resistant TLE who underwent surgery and who had a follow up of at least one year were entered into our study. They were from a series of patients (331 patients: 184 males, 147 females; mean age, 30.3 years; SD, 10.3; range, 662; mean age of epilepsy onset, 13.1 years; mean epilepsy duration, 16.2; SD, 10.2; range, 1-45) with medically refractory partial epilepsy for more than one year, referred to the epilepsy surgery unit of IRCCS NEUROMED, Pozzilli, Italy. All operated patients had undergone adequate trials of at least two first line and two add on drugs from among the new AEDs.

Abbreviations: AED, antiepileptic drugs; AMTL, anteromesial temporal lobectomy; Cl, confidence interval; DNET, dysembrioneuroepithelioma; EEG, electroencephalographic; ETL, extensive temporal lobectomy; ETLE, invasive presurgical evaluation; MRI, magnetic resonance imaging; MTS, mesial temporal sclerosis; Prot, protocol; TLE, temporal lobe epilepsy 


\section{Presurgical diagnostic protocol}

In our unit, the presurgical evaluation consists of a multilevel and individualised investigation characterised by noninvasive and invasive procedures. The invasive procedures (ETLE-Prot) are not the focus of our present study and will not be discussed.

\section{First diagnostic level}

All patients referred for surgery had a one day admission to the hospital to collect medical history data and perform preliminary neuroradiological and electroencephalographic (EEG) studies.

\section{Medical history}

A detailed medical history was obtained from all patients as a first step. Particular attention was paid to recognising the symptoms/signs at seizure onset and auras, because it is largely accepted in the literature that the initial seizure semiology usually provides valuable information about the seizure onset zone. ${ }^{14-16} \mathrm{~A}$ full clinical general and neurological examination was carried out in all patients.

\section{EEG monitoring}

The patients were monitored for 24 hours. Three 30 minute interictal EEG "standard" samples in the awake state, including hyperventilation and photic stimulation, and all sleep recordings were evaluated to assess the presence of background abnormalities, interictal slow activity, and epileptiform activity (focal (over one to three channels), regional (over three or more channels), hemispheric (over all channels of one side), and diffuse (over all channels of both sides) $).{ }^{17}$

\section{Neuroradiological evaluation}

The patients underwent brain 1.5 Tesla magnetic resonance imaging (MRI) examination and, in selected cases, brain computed tomography scans. The MRI scans were reviewed by a neuroradiologist, experienced in the field of epilepsy, masked to the clinical and outcome data. At review, MRI data were classified as follows: mesial temporal sclerosis (MTS) or lesion (low grade tumours, dysembrioneuroepithelioma (DNET), other tumour, dysplasia, or other). The presence of MTS was evaluated qualitatively by visual inspection of MRI: atrophy (on Tl weighted sequences) and increased mesial temporal signal intensity (on $\mathrm{T} 2$ weighted and fluid attenuated inversion recovery sequences) were considered markers of MTS. Volumetry was not performed. ${ }^{18} 19$

\section{Second diagnostic level}

The patients admitted to the second diagnostic level were evaluated by longterm video-EEG monitoring. They also underwent a comprehensive neuropsychological and psychiatric assessment, the results of which are beyond the scope of this paper and will be reported elsewhere.

The video-EEG recording technique ${ }^{20}$ was performed with collodion fixed scalp electrodes (16 EEG channels, positioned according to international 10-20 system, and one electrocardiographic channel to monitor ictal heart rate findings). ${ }^{21}$ All patients admitted to video-EEG monitoring had at least one seizure recorded.

\section{Ictal EEG findings}

We categorised ictal events as seizures with subjective phenomena only (auras) and seizures with symptoms and/ or signs, with or without loss of contact (simple partial or complex partial seizures).

Each ictal event was correlated with the corresponding EEG changes, when present.

According to the site, we distinguished between focal, regional, hemispheric, or diffuse discharges.

According to the EEG seizure pattern appearance and course, ictal EEG changes were classified into ictal onset pattern (first sudden change of frequency with attenuation or appearance of a new rhythm), ictal core pattern, late patterns, and post ictal pattern.

In patients with TLE, we used a classification of EEG ictal core patterns according to well known studies ${ }^{22-24}$ concerning ictal scalp-intracranial recordings, which showed a strict correlation between ictal scalp morphology of discharge and different temporal lobe structures involved at seizure onset, as follows:

- Type 1: antero-temporal 5-9 Hz discharge, associated with a highly probable onset in the mesial temporal structures.

- Type 2: temporal 2-4 Hz discharge, associated with a highly probable onset in the lateral temporal structures.

EEG ictal onset in extratemporal regions excluded the patients from the TLE-Prot.

\section{Ictal clinical findings}

In selecting the patients with TLE, ictal onset semiology characterised by focal motor, sensory, visual phenomena, or complex motor manifestations (forced head turning or hypermotor behaviour) suggesting a non-temporal lobe seizure onset, led to exclusion from the TLE-Prot.

In implementing the TLE diagnostic grid, despite the fact that no ictal behaviour is specific for temporal lobe seizures, we considered some initial symptoms/signs as highly suggestive of temporal seizures and classified them into a mesial or lateral cluster (table 1).

\section{TLE protocol (fig 1)}

The first diagnostic step in selecting patients to admit to the TLE-Prot was the evaluation of the MRI examination. If MRI showed an extratemporal lesion the patient was scheduled for the ETLE-Prot, whereas in all other cases the patient was admitted to the TLE-Prot (if other investigations performed in the first diagnostic level consistently pointed to TLE).

(A) If MRI showed a temporal tumour and seizure history was one to two years, the proposal of a lesionectomy

Table 1 Grid of anatomical, EEG, and clinical criteria used for the identification of TLE subsyndromes

\begin{tabular}{|c|c|c|}
\hline & Mesial cluster & Lateral cluster \\
\hline $\begin{array}{l}\text { Anatomical criteria } \\
\text { Ictal EEG criteria } \\
\text { Ictal clinical criteria }\end{array}$ & $\begin{array}{l}\text { Mesial temporal sclerosis or hippocampal atrophy and/or } \\
\text { medial structural lesion } \\
5-9 \mathrm{~Hz} \text { discharge, well localised to temporal regions } \\
\text { Rising epigastric aura, isolated or associated with other } \\
\text { vegetative symptoms } \\
\text { Early oroalimentary automatisms ("machonnement") } \\
\text { Dystonia of contralateral superior limb }\end{array}$ & $\begin{array}{l}\text { Focal atrophy of temporal lateral neocortex and/or lateral } \\
\text { structural lesion } \\
2-5 \mathrm{~Hz} \text { ictal discharge well localised to temporal regions } \\
\text { Psychic/experiential aura } \\
\text { Auditive or vestibular symptoms } \\
\text { Staring without oroalimentary automatisms }\end{array}$ \\
\hline
\end{tabular}




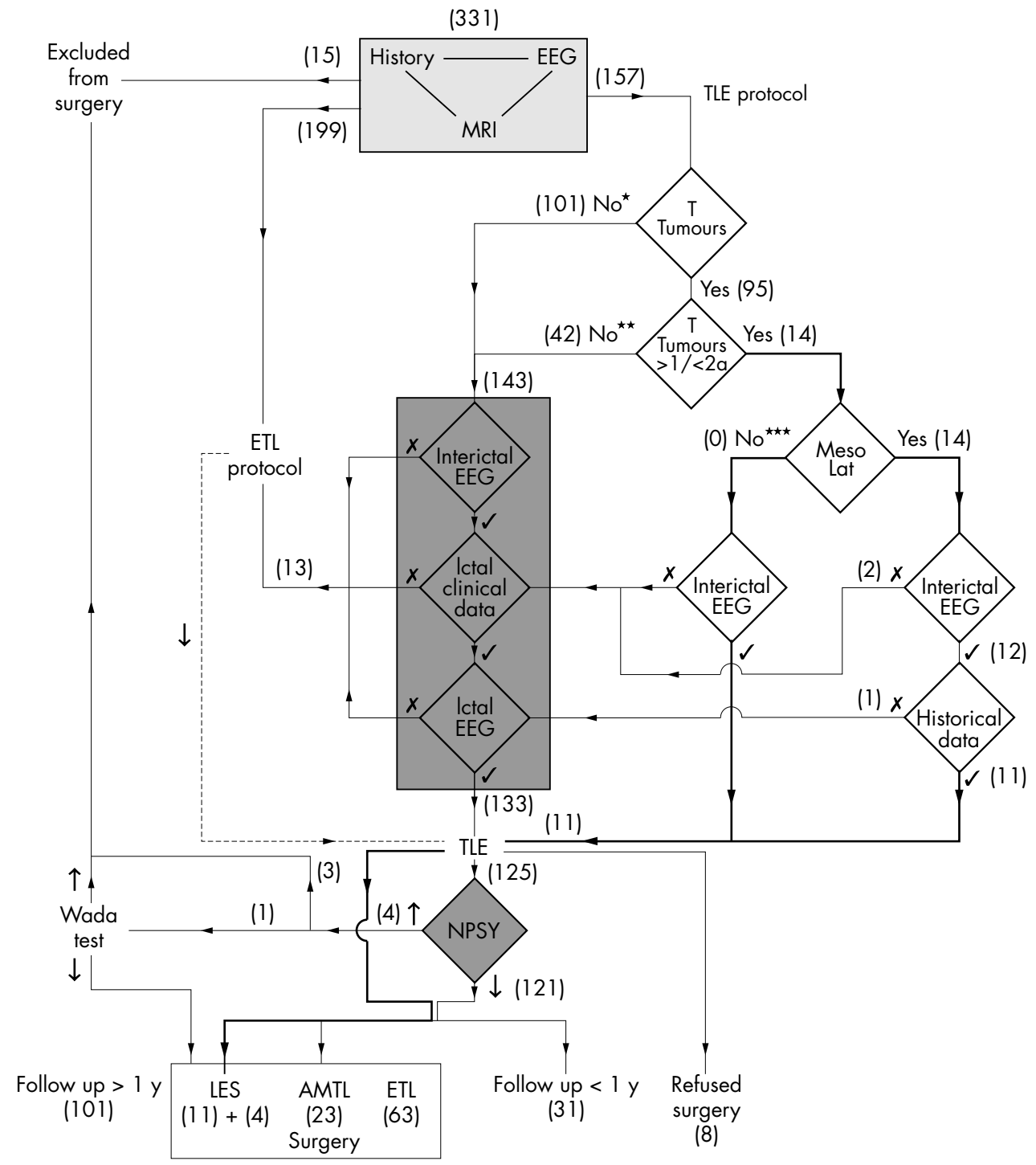

\begin{tabular}{|ll|}
\hline$\checkmark$ & Concordant for TLE \\
$x$ & Discordant for TLE \\
$\downarrow$ & Low risk for postoperative amnesia \\
$\uparrow$ & High risk for postoperative amnesia \\
$\star$ & HS, Dys, Atr, Neg \\
$\star \star$ & T tumours with seizure history $>2$ y \\
$\star \star \star$ & Mesio-lateral tumours \\
$\square$ & 1 st Diagnostic level \\
\hline$\square$ & 2nd Diagnostic level \\
- Fast protocol
\end{tabular}

Figure 1 Decisional algorithm for the diagnosis of TLE and surgical strategy. AMTL, anteromesial temporal lobectomy; Atr, atrophy; Dys, dysplasia; ET, extratemporal; ETL, extensive temporal lobectomy; HS, hippocampal sclerosis; I/Ictal, interictal; INV, invasive investigations; L, lateral; LES, lesionectomy; M, mesial; NPSY, neuropsycological assessment; TLE, temporal lobe epilepsy.

was evaluated without admission to level 2 diagnostic procedures: when the tumour involved mesiolateral structures and interictal EEG was concordant, and when the tumour involved mesial or lateral structures only and both interictal EEG and the patient and eyewitness history clinical data were concordant, a diagnosis of TLE was made and lesionectomy was offered (fast TLE-Prot); if interictal EEG and, in mesial or lateral tumours, clinical data were discordant, the patients were scheduled for the second diagnostic level.
(B) If MRI showed a temporal tumour and the seizure history was longer than two years, and in all other cases but extratemporal lesions, the patient was admitted to level 2 diagnostic procedures and underwent video-EEG. If both interictal and ictal electroclinical data revealed a diagnosis of TLE, surgery was offered and the operation was individualised according to the scheme for the identification of individualised TLE surgery. If interictal EEG and/or ictal electroclinical data were discordant, the patient was scheduled for the ETLE-Prot. 


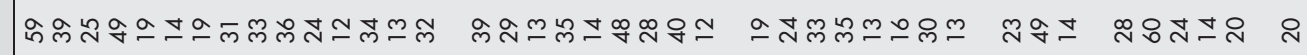

选 离

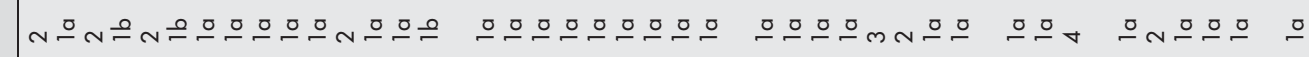

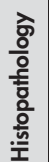

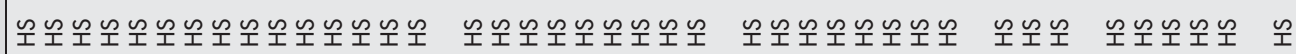

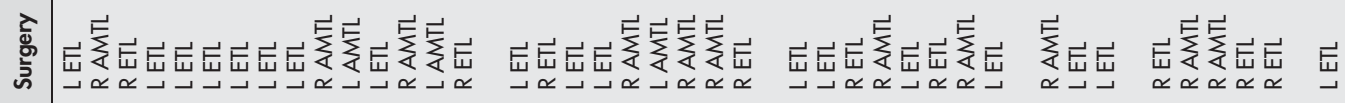

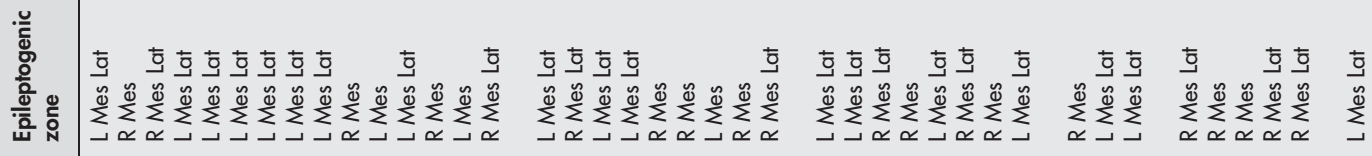

岃

혼 홍

욤

吾㐫

몬

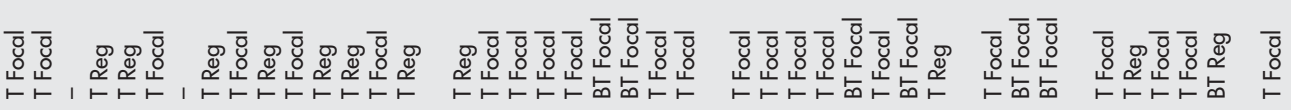

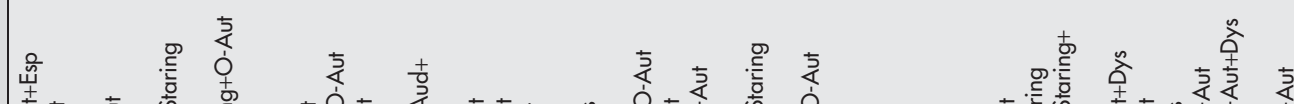

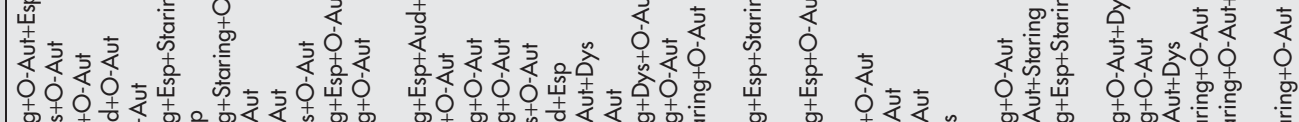

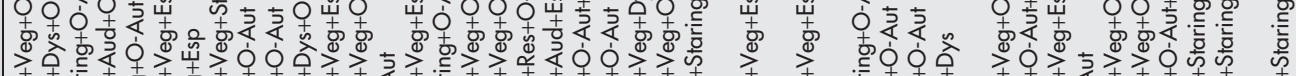

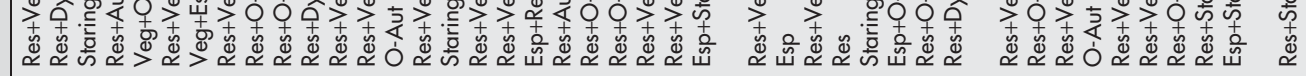

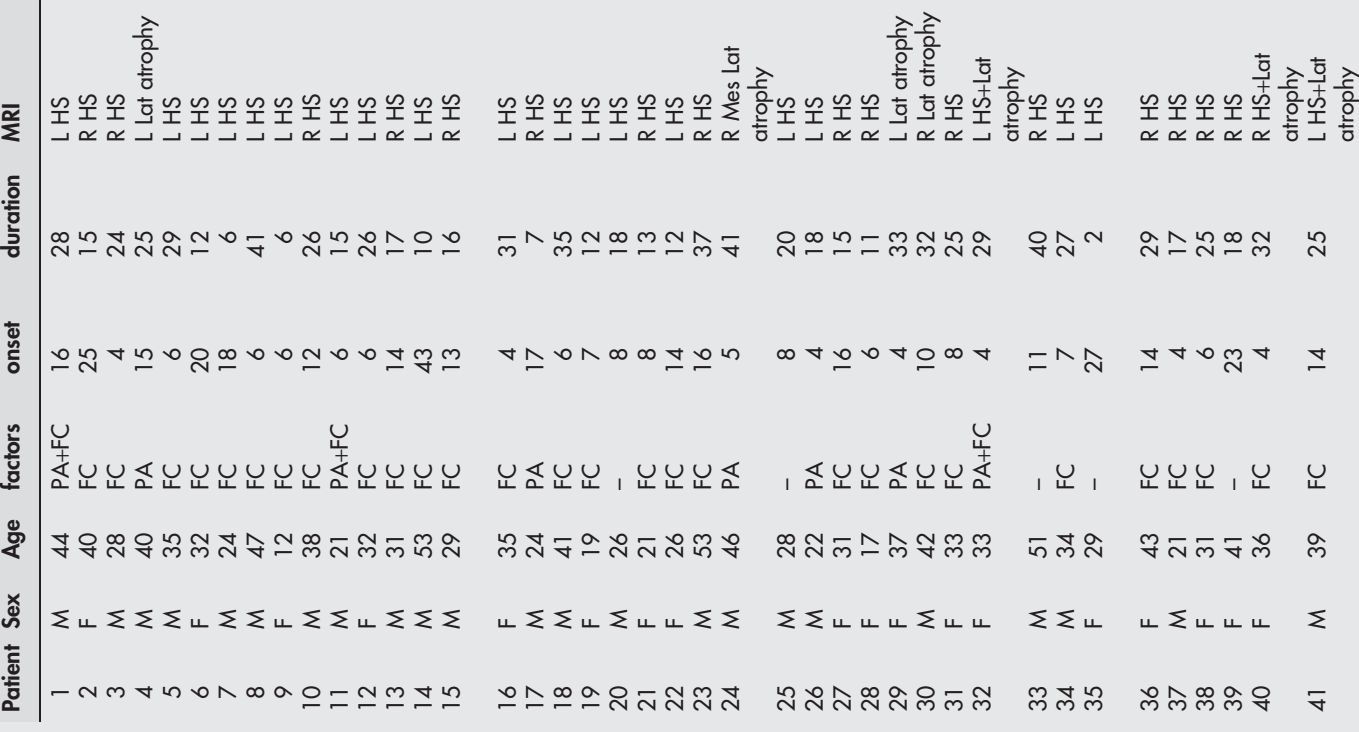




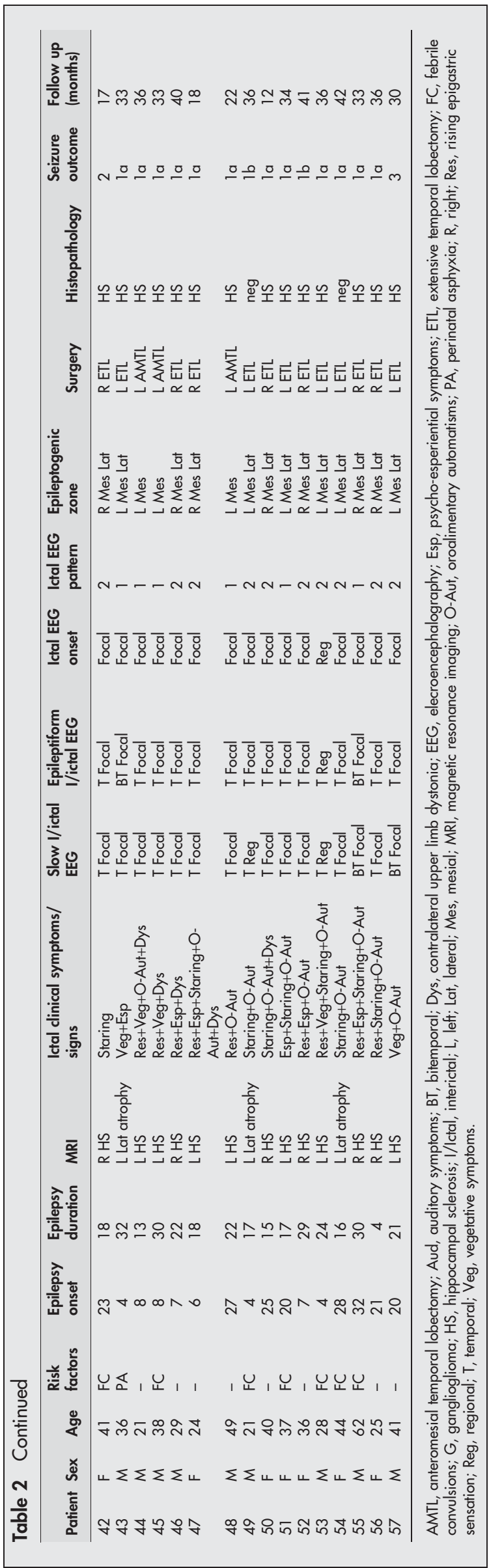

When neuropsychological data disclosed memory deficits contralateral to the side of the epileptogenic zone, suggesting a high risk for postoperative amnesia after temporal lobe surgery, the patient was scheduled for the Wada test or excluded from surgery.

Of 331 patients who underwent the preliminary assessment, 157 were scheduled for the TLE-Prot. MRI evaluation of these patients showed 84 hippocampal scleroses, 56 tumours (48 low grade neoplasms, eight cavernomas), 11 findings suggesting dysplasia (in four cases a hippocampal sclerosis was also evident), and four focal neocortical atrophies. In two patients, MRI showed no abnormalities. Among the 56 patients with MRI evidence of a tumour, 14 patients had a history of epilepsy for one to two years. In this group, in eight patients the lesion was located in the mesial structures and in six patients in the lateral aspects. No patient in this group had a lesion involving both the mesial and lateral structures. Three of 14 patients were excluded from a fast surgical protocol and were admitted to the second level because of non-concordance of EEG interictal or clinical data with MRI. In the 143 patients admitted to video-EEG monitoring, 929 seizures (mean number of seizures for each patient, 6.5; mean duration of monitoring days, 5.6) were recorded.

One hundred and fifty nine patients were admitted to the ETLE-Prot and 15 patients were excluded from surgery.

\section{Scheme for the identification of individualised TLE surgery}

To propose individualised and limited surgery strictly according to the epileptogenic zone, we developed a diagnostic grid (table 1) based on anatomical and ictal electroclinical criteria considered to be suggestive of epileptogenesis in the mesial or lateral temporal lobe. Patients were subdivided into the following three groups:

(1) Mesial TLE: when the anatomical criterion for mesial cluster, the EEG criterion for mesial cluster, and at least two of the clinical criteria for mesial cluster were met.

(2) Lateral TLE: when the anatomical criterion for lateral cluster, the EEG criterion for lateral cluster, and at least two of the clinical criteria for lateral cluster were met.

(3) Mesiolateral TLE: when at least one criterion for mesial cluster and at least one criterion for lateral cluster were met, or when the anatomical and the EEG criteria were concordant with one clinical criterion alone.

If brain imaging was negative, only the presence of EEG and clinical criteria were taken into account for the classification.

This scheme was used to define different surgical strategies: lesionectomy (fast TLE-Prot, tumorous lateral TLE), anteromesial temporal lobectomy (AMTL) in mesialTLE, and extensive temporal lobectomy (ETL) in hippocampal sclerosis/cryptogenic or lesional mesiolateral TLE.

\section{Surgery}

All operations were performed by the same epilepsy surgeon (VE).

Both ETL and AMTL included microsurgical resection of the amygdala and en bloc excision of the hippocampal formation and parahippocampal gyrus. These interventions differed in the extent of the neocortical resection. Nondominant ETL included excision of $4-4.5 \mathrm{~cm}$ of the superior temporal gyrus and the middle temporal gyrus, and 5-6 cm of the inferior temporal gyrus, whereas dominant ETL included excision of $4-5 \mathrm{~cm}$ of the middle and inferior temporal gyrus, although the superior gyrus was left intact. In AMTL, the extent of the neocortical excision was $3 \mathrm{~cm}$ for 
all the first three temporal gyri (sparing the superior gyrus in the dominant hemisphere). Lesionectomy consisted of complete removal of the foreign epileptogenic tissue alone.

\section{Seizure outcome assessment}

Seizure outcome was determined by the patient's report to the neurologist during the scheduled follow up visits, including 60 minutes awake EEG standard recordings, ${ }^{25}$ and it was classified according to Engel. ${ }^{26}$ During the first year of follow up, AEDs were kept constant in all patients.

\section{Statistical analysis}

Descriptive statistics were used to summarise the data for all variables. The confidence intervals (CI) for the proportion of seizure free patients in each group were calculated. The $\chi^{2}$ test was used to test for differences in outcome between patients with foreign tissue lesions and patients with hippocampal sclerosis.

\section{RESULTS}

A lesionectomy was offered to 11 patients with TLE after the fast TLE-Prot. After video-EEG monitoring, another 133 patients were diagnosed as having TLE, and 13 were admitted to the ETLE-Prot. Four of the 133 patients with TLE were excluded from surgery for psychiatric or neuropsychological disturbances. Therefore, surgery was offered to a total of 140 patients with TLE. Eight of these patients refused surgery.

Of the 132 consecutive patients with TLE who underwent surgery, 101 had a follow up of at least one year (range, 1260 months; mean duration, 30). No patients were lost to follow up, although 31 patients had a follow up period of less then one year and therefore were excluded from our study. All patients with TLE were divided in a "hippocampal sclerosis/cryptogenic" group (57 patients) (table 2) and a "tumours/cortical organisation disorders" group (44 patients) (table 3).

\section{Hippocampal sclerosis/cryptogenic group \\ General characteristics}

Fifty seven patients ( 25 females and 32 males; mean age, 33.9 years; SD, 10.2; range, 12-62; mean age of epilepsy onset, 12.4 years; SD, 8.7; range, 4-43; mean epilepsy duration, 21.5 years; SD, 9.5; range, 2-41) were classified into this group. Risk factors for epilepsy were found in 44 patients (febrile convulsions in 35 and perinatal asphyxia in nine patients).

Table 4 shows the results of presurgical investigations, type of operation and histopathology.

\section{Seizure outcome}

Forty seven (95\% CI, 70.1 to 91.2) patients were classified as Engel class I ( 42 as class Ia, five as Engel class Ib), seven as Engel class II, two as Engel class III, and one as Engel class IV.

\section{Tumours/cortical organisation disorders group General characteristics}

Forty four patients ( 21 females and 23 males; mean age, 32.3 years; SD, 12.7; range, 76-63; mean age of epilepsy onset, 19.7 years; SD, 14.7; range, $1-57$ years; mean epilepsy duration, 12.5 years; SD, 11.9; range, 1-45) were classified into this group. Risk factors for epilepsy were found in eight patients (febrile convulsions in five and perinatal asphyxia in three patients). Pathology showed 21 low grade tumours, eight DNETs, five cavernous angiomas, nine cortical dysplasias, and one focal Rasmussen encephalitis.

Table 4 shows the results of the presurgical investigations, the type of surgery, and the histopathology.

\section{Seizure outcome}

Thirty nine (95\% CI, 75.4 to 96.2) patients were classified as Engel class I ( 36 as Engel class Ia, three as Engel class Ib) and five as Engel class II.

\section{Morbidity}

Permanent postoperative complications occurred in two patients $(2 \%)$ : one patient developed a hemiplegia after ETL, whereas the other developed a hemianopia after lateral lesionectomy.

With regard to the neuropsychological outcome, no patients suffered from serious or clinically evident neuropsychological morbidity at the one year follow up assessment.

The seizure outcome of patients with foreign tissue lesions (39 in Engel class 1) was not significantly different from that of patients with hippocampal sclerosis (47 in Engel class 1) $\left(\chi^{2}=0.75 ; p=0.39\right)$.

\section{DISCUSSION}

TLE is the "model" surgically remediable epileptic syndrome, first because it is the most frequent among focal epilepsies, and second because it is often resistant to medical treatment. Moreover, because TLE often reflects epileptogenesis localised in "discrete" and anatomically well circumscribed structures (the amygdalo-hippocampal complex), whose radiological and electroclinical seizure correlates are well known, it may be considered particularly eligible for surgical treatment. ${ }^{5627}$ Therefore, almost all centres offer surgery for TLE after a noninvasive diagnostic investigation, reporting good seizure outcome and surgery safety. However, common guidelines regarding the relative weight of the different presurgical investigations are still not well established. As a consequence, several protocols emphasise neuroimaging (MRI), ${ }^{28}$ whereas other protocols emphasise video-EEG recording. ${ }^{29}$ Few studies have focused on the problem of implementing a "reproducible" methodology to allow TLE surgery with an optimal cost-benefit ratio, both in terms of seizure outcome/ quality of life and costs. ${ }^{29}$ However, even in the best published TLE surgical series, only $80 \%$ of patients have "good outcome", often including patients in Engel class II who are not seizure free. ${ }^{4}$ Moreover, several non-invasive protocols include very expensive procedures, such as MRI volumetric analysis, functional MRI, single photon emission computed tomography, positron emission tomography, and the Wada test. This reduces the cost effectiveness of surgery for epilepsy and the number of patients to whom it can be offered..$^{28-31}$

Another open issue concerns the identification, by means of non-invasive techniques, of different TLE subsyndromes (mesial, lateral, and mesiolateral TLE) ${ }^{32}$ so that different surgical strategies can be offered as appropriate. In fact, the "classic" surgical approach in TLE tends to consider standard surgical procedures (temporal lobectomy or selective amygdalohippocampectomy), ${ }^{29334}$ with the risk of removing cortical regions not involved in epileptogenesis or not resecting the whole epileptogenic zone.

When MRI shows a tumour with a brief (less than two years) seizure history, we consider ictal video-EEG recording unnecessary when the patient and eyewitness history, clinical semiology, and/or interictal EEG are concordant with the tumour site. ${ }^{35}$ Recurrent seizures are recognised as a potential cause of neuronal damage, and hence have been hypothesised to modify the extent of the epileptogenic zone in time. ${ }^{36}$ Therefore, in tumorous epilepsy with a brief history, it is more likely that the epileptogenic zone is restricted to the lesion, and surgery may be limited to lesionectomy alone.

The results of lesionectomy alone for the treatment of TLE as a result of a temporal lobe mass lesion have been controversial. Jooma and colleagues ${ }^{37}$ and Cascino and colleagues $^{38}$ reported that lesionectomy led to a "seizure 


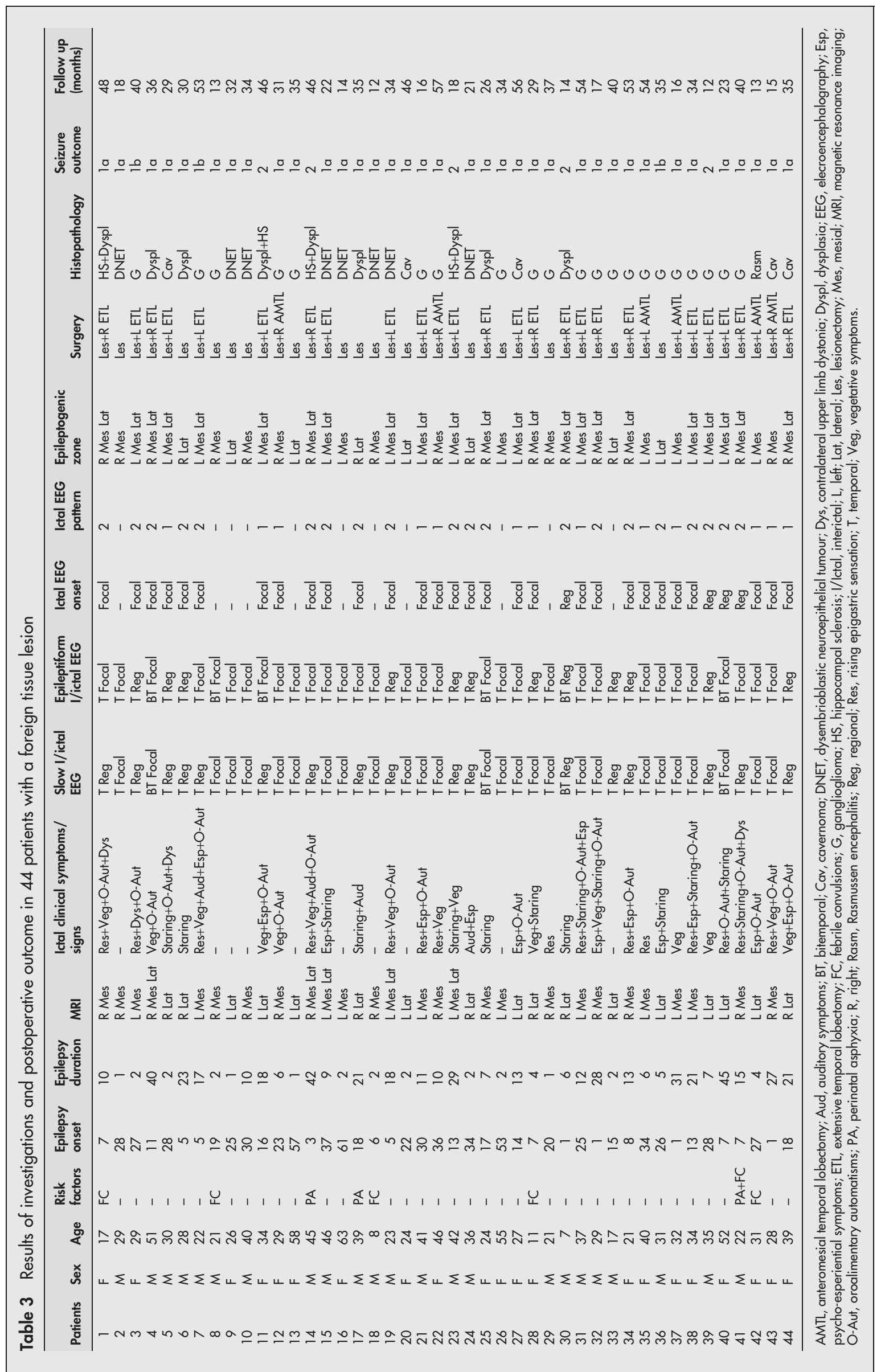


Table 4 Global results of presurgical evaluation, type of surgery, and histopathology in the 101 operated patients with follow up after surgery $>1$ year

\begin{tabular}{|c|c|c|c|c|}
\hline & \multicolumn{2}{|c|}{$\begin{array}{l}\text { Hippocampal } \\
\text { sclerosis/ } \\
\text { cryptogenic group }\end{array}$} & \multicolumn{2}{|c|}{$\begin{array}{l}\text { Tumorous/ } \\
\text { COD group }\end{array}$} \\
\hline & $\overline{\mathrm{N}}$ & (\%) & $\bar{N}$ & $(\%)$ \\
\hline Interictal EEG abnormalities & 55 & 95.6 & 44 & 100 \\
\hline Focal & 34 & 61.8 & 24 & 54.5 \\
\hline Regional & 12 & 21.8 & 16 & 36.4 \\
\hline Bitemporal & 9 & 16.4 & 4 & 9.1 \\
\hline Epileptiform abnormalities & 56 & 98.2 & 44 & 100 \\
\hline Focal & 37 & 66.1 & 27 & 61.4 \\
\hline Regional & 4 & 7.1 & 11 & 25 \\
\hline Bitemporal & 15 & 26.8 & 6 & 13.6 \\
\hline \multicolumn{5}{|l|}{ Ictal clinical findings } \\
\hline Rising epigastric sensation & 41 & 71.9 & 16 & 36.4 \\
\hline Other vegetative symptoms & 23 & 40.3 & 16 & 36.4 \\
\hline Psyco-experential phenomena & 19 & 33.3 & 13 & 29.5 \\
\hline Auditory symptoms & 3 & 5.3 & 4 & 9.1 \\
\hline Oroalimentary automatisms & 21 & 36.8 & 14 & 31.8 \\
\hline Staring & 46 & 80.7 & 20 & 45.4 \\
\hline Controlateral dystonia & 13 & 22.8 & 4 & 9.1 \\
\hline \multicolumn{5}{|l|}{ Ictal EEG findings } \\
\hline Focal & 52 & 91.2 & 29 & 65.9 \\
\hline Regional & 5 & 8.8 & 4 & 9.1 \\
\hline Pattern type 1 & 26 & 45.6 & 13 & 29.5 \\
\hline Pattern type 2 & 31 & 54.4 & 20 & 45.4 \\
\hline \multicolumn{5}{|l|}{ Epileptogenic zone } \\
\hline Mesial & 17 & 29.9 & 13 & 29.5 \\
\hline Lateral & - & - & 8 & 18.1 \\
\hline Mesiolateral & 40 & 70.1 & 23 & 52.2 \\
\hline \multicolumn{5}{|l|}{ Surgery } \\
\hline ETL & 40 & 70.2 & - & - \\
\hline AMTL & 17 & 29.8 & - & - \\
\hline ETL+lesionectomy & - & - & 23 & 52.3 \\
\hline AMTL+lesionectomy & - & - & 6 & 13.6 \\
\hline Lesionectomy & - & - & 15 & 34.1 \\
\hline \multicolumn{5}{|l|}{ Histopathology } \\
\hline MTS & 55 & 96.5 & - & - \\
\hline $\begin{array}{l}\text { Low grade tumours } \\
\text { (ganglioglioma, diffuse } \\
\text { astrocytoma) }\end{array}$ & - & - & 21 & 47.7 \\
\hline DNET & - & - & 8 & 18.2 \\
\hline Cavernoma & - & - & 5 & 11.4 \\
\hline Dysplasia & - & - & $\begin{array}{l}9 \\
\text { (+4 MTS) }\end{array}$ & 20.5 \\
\hline Focal Rasmussen encephalitis & - & - & 1 & 2.2 \\
\hline Negative & 2 & 3.5 & - & - \\
\hline \multicolumn{5}{|c|}{$\begin{array}{l}\text { AMTL, anteromesial temporal lobectomy; COD, cortical organisation } \\
\text { disorders; DNET, dysembrioneuroepithelioma; EEG, } \\
\text { electroencephalographic; ETL, extensive temporal lobectomy; MTS, } \\
\text { mesial temporal sclerosis. }\end{array}$} \\
\hline
\end{tabular}

free" condition in only $20 \%$ of patients with temporal lobe mass lesions, whereas Fried and colleagues $^{39}$ reported a seizure free outcome in $80 \%$ of patients. ${ }^{35}$ Kraemer et al reported that $73 \%$ of the patients affected by TLE as a result of vascular malformations were seizure free (no seizures in the last year of follow up, according to Dukes's classification ${ }^{40}$ after lesionectomy). ${ }^{35}$ In general, it is agreed that if the seizure disorder exists for more than a year before surgical resection, lesionectomy alone may not be efficacious. ${ }^{374}$ Other studies ${ }^{42}{ }^{43}$ corroborate the assumption that a shorter seizure disorder duration correlates with a better prognosis when lesionectomy is the surgical approach.

In our series, 14 patients with TLE had a short seizure history and MRI evidence of a temporal mass lesion: three of these patients were excluded from "fast" lesionectomy and were admitted to diagnostic level 2 for video-EEG recording because of lesional pathology not colocalised with EEG interictal findings, whereas 11 patients had lesionectomy with excellent outcome (all in Engel class la). Two of the three abovementioned patients who were entered into diagnostic level 2 had extensive temporal lobectomy (one in
Engel class la and one in Engel class lb), whereas the other patient had lesionectomy (Engel class la).

Although some studies ${ }^{44}$ report that ictal video-EEG recording may be not mandatory in patients with TLE, we decided to admit most lesional or non-lesional patients with TLE to video-EEG recording. This strategy is obviously "obligatory" in patients with normal MRI or absent or bilateral interictal EEG epileptiform abnormalities. Moreover, in our opinion, a tailored operation cannot be offered without having ictal EEG and clinical data, which allow the three different TLE subsyndromes to be identified. Therefore, we developed a diagnostic grid for TLE surgery based on correlations among anatomical, electrical, and clinical data, in accordance with principles first planned by Bancaud and Talairach, ${ }^{9}$ and later developed by their successors, ${ }^{10-12}$ obtained by non-invasive investigations. According to many studies that evaluated the localising value of a single diagnostic criterion, ${ }^{22}{ }^{23}{ }^{45-52}$ the data obtained from presurgical evaluation were grouped in clusters strongly suggesting the location of epileptogenesis in the mesial or lateral aspect of the temporal lobe. Then, based on our experience, specific combinations of different criteria were used for localisation. Although most authors agree about the localising value of clinical signs and of neuroradiological findings in suggesting the epileptogenic zone, to our knowledge, only a few clinical studies $^{53}$ have used EEG ictal patterns for localising purposes. In our classification scheme, we particularly stressed the localising value of the ictal scalp EEG pattern in TLE, according to the theories of Pacia and Ebersole, ${ }^{23}$ based on "the most comprehensive systematic study of scalp-intracranial ictal EEG findings in patients with temporal lobe epilepsy". ${ }^{54}$ Their findings confirmed and extended previous studies on this issue, ${ }^{55} 56$ revealing that $>5 \mathrm{~Hz}$ ictal EEG pattern over the temporal regions is the characteristic pattern associated with mesial temporal seizures. In contrast, there is comparatively little information about neocortical temporal lobe seizures, and in most cases the available descriptions are shown in negative terms, emphasising the absence of features typical of mesial temporal lobe seizures. In light of this, the studies of Ebersole's and Foldvary's groups should still be thought of as important contributions; moreover, they depicted neocortical temporal lobe seizures in positive terms. Both authors agree that low frequency scalp EEG ictal patterns in temporal lobe seizures are highly related to the lateral neocortical seizure onset zone. Compared with other non-invasive diagnostic protocols ${ }^{31357}$ for TLE surgery, aimed at selecting those patients who should benefit from standard temporal lobectomy and identifying those patients who should be investigated invasively or excluded, our diagnostic grid enables TLE classification to be refined into the mesial, temporal, and mesiotemporal subtypes. Hence, surgery can be specifically planned, as far as possible, according to the extension of the epileptogenic zone in the three different TLE subtypes (that is, AMTL in mesial-TLE, ETL in mesiolateral-TLE, and lesionectomy in lesional lateralTLE), avoiding unnecessarily extensive surgery. As suggested by studies based on stereotactic intracerebral EEG recordings, ${ }^{58}$ during which the simultaneous involvement of both the amygdala, the hippocampus, and the temporal pole at the onset of temporal lobe seizures was often observed, the so called "mesial" temporal lobe seizures probably do not always arise solely from the amygdalo-hippocampo-parahippocampal complex. Therefore, in the absence of intracerebral recordings, in mesial-TLE, we decided to use the AMTL resection, which includes the temporal pole in addition to the mesial temporal structures, rather than the selective amygdalo-hippocampectomy.

The usefulness of our T-Prot was confirmed by the excellent outcome (overall, $85.1 \%$ in Engel class 1), although 
we cannot exclude the possibility that a similar outcome would have been achieved without using a tailored resection. Given that the diagnostic approach we describe is based on a global assessment that correlates and combines a set of criteria, we could not carry out an analysis of the performance of each diagnostic criterion (anatomical, ictal EEG, and ictal clinical) in terms of sensitivity and specificity. In our classification method, it is not possible to assign a patient to a group using single criteria, and this made it impossible for us to test the performance of a single criterion. In any case, the performance of the full set of criteria was very satisfactory.

Although our findings need to be confirmed by further studies with a longer follow up duration and by randomised controlled trials comparing our protocol with other established protocols, they suggest that in most patients with TLE different subsyndromes can be accurately identified using non-invasive anatomo-electro-clinical data, and can be treated effectively and safely with a tailored operation.

\section{ACKNOWLEDGEMENTS}

We thank the nursing staff and the team of neurophysiology technicians (L Cacciola, F Orabona, and R Ponticelli) who performed meticulous video-EEG recordings in all patients.

\section{Authors' affiliations \\ P P Quarato, G Di Gennaro, A Mascia, L G Grammaldo, G N Meldolesi, T Giampà, C Falco, F Sebastiano, M Manfredi, G Cantore, V Esposito, Department of Neurological Sciences, Epilepsy Surgery Unit, IRCCS Neuromed, Pozzilli (IS), Italy \\ P Onorati, Department of Human Physiology and Pharmacology, University "La Sapienza", Rome, Italy \\ A Picardi, Centre of Epidemiology and Health Surveillance and Promotion, Italian National Institute of Health, Rome, Italy \\ Competing interests: none declared}

\section{REFERENCES}

1 Beghi E, Garattini L, Ricci E, EPICOS Group, et al. Direct cost of medical management of epilepsy among adults in Italy: a prospective cost-of-illness study (EPICOS). Epilepsia 2004;45:171-8.

2 Rosenow F, Luders H. Presurgical evaluation of epilepsy. Brain $2001 ; 124: 1683-700$

3 Engel J Jr. Surgery for seizures. N Engl J Med 1996;334:647-52.

4 Engel J Jr. Finally, a randomized, controlled trial of epilepsy surgery NEngl J Med 2001;345:365-7.

5 Wiebe S, Blume WT, Girvin JP, et al. Effectiveness and efficiency of surgery for temporal lobe epilepsy study group. A randomized, controlled trial of surgery for temporal-lobe epilepsy. N Engl J Med 2001;345:31 1-18.

6 Engel J Jr, Williamson PD, Wieser HG. Mesial temporal lobe epilepsy. In: Engel J Jr, Pedley J, eds. Epilepsy: a comprehensive textbook. New York: Raven Press, 1997:2417-26.

7 Engel J Jr, Cascino GD, Shields WD. Surgical remediable syndromes. In: Engel J Jr, Pedley J, eds. Epilepsy: a comprehensive textbook. New York: Raven Press, 1997:1687-96.

8 Engel J Jr. Principles of epilepsy surgery. In: Shorvon S, Dreifuss F, Fish D, et al, eds. The treatment of epilespy. Oxford: Blackwell Sciences, 1996:519-29.

9 Bancaud J, Talairach J, Bonis A, et al. La Stéréo-électroencéphalographie dans l'épilepsie. Paris: Masson, 1965

10 Munari C, Tassi L, Lo Russo G, et al. La terapia chirurgica nelle epilessie parziali: schematizzazione metodologica o approccio personalizzato. Bollettino della Lega Italiana Contro L'Epilessia 1990;70/1:61-7.

11 Bartolomei F, Guye M, Gavaret $M$, et al. The presurgical evaluation of epilepsies. Rev Neurol (Paris) 2002; 158(5 Pt 2):4S55-64.

12 Chauvel P, Halgren E. Les illusions et le hallucinations complexes dans le crises du lobe temporal. In: Crises epileptiques et epilepsies du lobe temporal, Vol. I. Gentilly: Documentation Medicale Labaz, 1991:113-24.

13 Bartolomei F, Wendling F, Vignal JP, et al. Seizures of temporal lobe epilepsy: identification of subtypes by coherence analysis using stereo-electroencephalography. Clin Neurophysiol 1999;110:1741-54.

14 Luders HO, Awad IA. Conceptual considerations. In: Luders HO, ed. Epilepsy surgery. New York: Raven Press, 1992:51-62.

15 Palmini A, Gloor P. The localizing value of auras in partial epilepsies. Neurology 1992;42:801-8.

16 Henkel A, Noachtar S, Pfander M, et al. The localizing value of the abdominal aura and its evolution. Neurology 2002;58:271-6.

17 Di Gennaro G, Quarato PP, Onorati P, et al. Localizing significance of temporal intermittent rhythmic delta activity (TIRDA) in drug-resistant focal epilepsy. Clin Neurophysiol 2003;114:70-8.
18 Clusmann H, Schramm J, Kral T, et al. Prognostic factors and outcome after different types of resection for temporal lobe epilepsy. J Neurosurg 2002;97:1131-41.

19 Garcia PA, Laxer KD, Barbaro NM, et al. Prognostic value of qualitative magnetic resonance imaging hippocampal abnormalities in patients undergoing temporal lobectomy for medically refractory seizures. Epilepsia 1994;35:520-4.

20 American Electroencephalography Society. Guidelines for long-term monitoring for epilepsy. J Clin Neurophysiol 1986;63:107-11.

21 Di Gennaro G, Quarato PP, Sebastiano F, et al. Ictal heart rate increase precedes EEG discharge in drug-resistant mesial temporal lobe seizures. Clin Neurophysiol 2004;115:1169-77.

22 Ebersole JS, Pacia SV. Localization of temporal lobe foci by ictal EEG patterns. Epilepsia 1996;37:386-99.

23 Pacia SV, Ebersole JS. Intracranial EEG substrates of scalp ictal patterns from temporal lobe foci. Epilepsia 1997;38:642-54

24 Ebersole JS. Localization of epileptic foci in temporal lobe epilepsy. Electroencephalogr Clin Neurophysiol 1997;103:47.

25 Di Gennaro G, Quarato PP, Sebastiano F, et al. Postoperative EEG and seizure outcome in temporal lobe epilepsy surgery. Clin Neurophysiol 2004; 115:1212-19

26 Engel J Jr. Outcome with respect to epileptic seizures. In: Engel J Jr, ed. Surgical treatment of the epilepsies. New York: Raven Press, 1987:553-71.

27 Lowe AJ, David E, Kilpatrick CJ, et al. Epilepsy surgery for pathologically proven hippocampal sclerosis provides long-term seizure control and improved quality of life. Epilepsia 2004;45:237-42.

28 Kilpatrick C, Cook M, Kaye A, et al. Non-invasive investigations successfully select patients for temporal lobe surgery. I Neurol Neurosurg Psychiatry 1997;63:327-33

29 Sperling MR, O'Connor MJ, Saykin AJ, et al. A non-invasive protocol for anterior temporal lobectomy. Neurology 1992;42:416-22.

30 Salanova V, Markand O, Worth R. Temporal lobe epilepsy: analysis of patients with dual pathology. Acta Neurol Scand 2004;109:126-31.

31 Akanuma N, Koutroumanidis $M$, Adachi N, et al. Presurgical assessment of memory-related brain structures: the Wada test and functional neuroimaging. Seizure 2003;12:346-58.

32 Bartolomei F, Wendling F, Vignal JP, et al. Seizures of temporal lobe epilepsy: identification of subtypes by coherence analysis using stereo-electroencephalography. Clin Neurophysiol 1999;1 10:1741-54.

33 Wieser HG. Selective amygdalo-hippocampectomy for temporal lobe epilepsy. Epilepsia 1988;29(suppl 2):S100-13.

34 Kirkpatrick PJ, Honavar M, Janota I, et al. Control of temporal lobe epilepsy following en bloc resection of low-grade tumors. J Neurosurg 1993;78:19-25.

35 Kraemer DL, Griebel ML, Lee N, et al. Surgical outcome in patients with epilepsy with occult vascular malformations treated with lesionectomy. Epilepsia 1998;39:600-7.

36 Kalviainen R, Salmenpera T. Do recurrent seizures cause neuronal damage? A series of studies with MRI volumetry in adults with partial epilepsy. Prog Brain Res 2002;135:279-95.

37 Jooma $\mathbf{R}$, Yeh HS, Privitera MD, et al. Lesionectomy versus electrophysiologically guided resection for temporal lobe tumors manifesting with complex partial seizures. J Neurosurg 1995;83:231-6.

38 Cascino GD, Kelly PJ, Sharbrough FW, et al. Long-term follow-up of stereotactic lesionectomy in partial epilepsy: predictive factors and electroencephalographic results. Epilepsia 1992;33:639-44.

39 Fried I, Kim JH, Spencer DD. Hippocampal pathology in patients with intractable seizures and temporal lobe masses. J Neurosurg 1992;76:735-40

40 Walczak TS, Radtke RA, McNamara JO, et al. Anterior temporal lobectomy for complex partial seizures: evaluation, results, and long-term follow-up in 100 cases. Neurology 1990;40(3 Pt 1):413-18

41 Cohen DS, Zubay GP, Goodman RR. Seizure outcome after lesionectomy for cavernous malformations. J Neurosurg 1995;83:237-42.

42 Morris HH, Estes ML, Gilmore R, et al. Chronic intractable epilepsy as the only symptom of primary brain tumor. Epilepsia 1993;34:1038-43.

43 Luyken C, Blumcke I, Fimmers R, et al. The spectrum of long-term epilepsyassociated tumors: long-term seizure and tumor outcome and neurosurgical aspects. Epilepsia 2003;44:822-30.

44 Cendes F, Li LM, Watson C, et al. Is ictal recording mandatory in temporal lobe epilepsy? Not when the interictalelectroencephalogram and hippocampal atrophy coincide. Arch Neurol 2000;57:497-500.

45 Williamson PD, French JA, Thadani VM, et al. Characteristics of medial temporal lobe epilepsy: II. Interictal and ictal scalp electroencephalography, neuropsychological testing, neuroimaging, surgical results, and pathology. Ann Neurol 1993;34:781-7.

46 O'Brien TJ, Kilpatrick C, Murrie V, et al. Temporal lobe epilepsy caused by mesial temporal sclerosis and temporal neocortical lesions. A clinical and electroencephalographic study of 46 pathologically proven cases. Brain 1996; 1 19:2133-41.

47 Bleasel A, Kotagal P, Kankirawatana P, et al. Lateralizing value and semiology of ictal limb posturing and version in temporal lobe and extratemporal epilepsy. Epilepsia 1997;38:168-74.

48 Foldvary N, Lee N, Thwaites G, et al. Clinical and electrographic manifestations of lesional neocortical temporal lobe epilepsy. Neurology 1997;49:757-63

49 Mohamed A, Wyllie E, Ruggieri P, et al. Temporal lobe epilepsy due to hippocampal sclerosis in pediatric candidates for epilepsy surgery. Neurology 2001;56:1643-9.

50 Foldvary N, Klem N, Hammel J, et al. The localizing value of ictal EEG in focal epilepsy. Neurology 2001;57:2022-8. 
51 Gil-Nagel A, Risinger MW. Ictal semiology in hippocampal versus extrahippocampal temporal lobe epilepsy. Brain 1997;120:183-92.

52 Moriarity JL, Boatman D, Krauss GL, ef al. Human "memories" can be evoked by stimulation of the lateral temporal cortex after ipsilateral medial temporal lobe resection. J Neurol Neurosurg Psychiatry 2001;71:549-51.

53 Garcia M, D'Giano C, Estelles S, et al. Ictal tachycardia: its discriminating potential between temporal and extra-temporal seizure foci. Seizure 2001;10:415-19.

54 Sadler M, Desbiens R. Scalp EEG in temporal lobe epilepsy surgery. Can J Neurol Sci 2000;27(suppl 1):S22-8, discussion S50-2.
55 Risinger MW, Engel J Jr, Van Ness PC et al. Ictal localization of temporal lobe seizures with scalp/sphenoidal recordings. Neurology 1989;39:1288-93.

56 Walczak TS, Radtke RA, Lewis DV. Accuracy and interobserver reliability of scalp ictal EEG. Neurology 1992;42:2279-85.

57 Thadani VM, Williamson PD, Berger R, et al. Successful epilepsy surgery without intracranial EEG recording: criteria for patient selection. Epilepsia 1995;36:7-15.

58 Kahane P, Chabardes S, Minotti L, et al. The role of the temporal pole in the genesis of temporal lobe seizures. Epileptic Disord 2002:4(suppl 1):S51-8.

\section{Clinical Evidence - Call for contributors}

Clinical Evidence is a regularly updated evidence-based journal available worldwide both as a paper version and on the internet. Clinical Evidence needs to recruit a number of new contributors. Contributors are healthcare professionals or epidemiologists with experience in evidence-based medicine and the ability to write in a concise and structured way.

Areas for which we are currently seeking authors:

- Child health: nocturnal enuresis

- Eye disorders: bacterial conjunctivitis

- Male health: prostate cancer (metastatic)

- Women's health: pre-menstrual syndrome; pyelonephritis in non-pregnant women

However, we are always looking for others, so do not let this list discourage you.

Being a contributor involves:

- Selecting from a validated, screened search (performed by in-house Information Specialists) epidemiologically sound studies for inclusion.

- Documenting your decisions about which studies to include on an inclusion and exclusion form, which we keep on file.

- Writing the text to a highly structured template (about 1500-3000 words), using evidence from the final studies chosen, within 8-10 weeks of receiving the literature search.

- Working with Clinical Evidence editors to ensure that the final text meets epidemiological and style standards.

- Updating the text every six months using any new, sound evidence that becomes available. The Clinical Evidence in-house team will conduct the searches for contributors; your task is simply to filter out high quality studies and incorporate them in the existing text.

- To expand the topic to include a new question about once every 12-18 months.

If you would like to become a contributor for Clinical Evidence or require more information about what this involves please send your contact details and a copy of your CV, clearly stating the clinical area you are interested in, to Klara Brunnhuber (kbrunnhuber@ bmigroup.com).

\section{Call for peer reviewers}

Clinical Evidence also needs to recruit a number of new peer reviewers specifically with an interest in the clinical areas stated above, and also others related to general practice. Peer reviewers are healthcare professionals or epidemiologists with experience in evidence-based medicine. As a peer reviewer you would be asked for your views on the clinical relevance, validity, and accessibility of specific topics within the journal, and their usefulness to the intended audience (international generalists and healthcare professionals, possibly with limited statistical knowledge). Topics are usually 1500-3000 words in length and we would ask you to review between 2-5 topics per year. The peer review process takes place throughout the year, and our turnaround time for each review is ideally 10-14 days.

If you are interested in becoming a peer reviewer for Clinical Evidence, please complete the peer review questionnaire at www.clinicalevidence.com or contact Klara Brunnhuber (kbrunnhuber@bmigroup.com). 